\title{
Enhancement of the yield of long helical structure of recombinant nucleocapsid protein of Newcastle disease virus
}

\begin{abstract}
A deletion mutant of the nucleocapsid protein (NPq 375) of Newcastle disease virus selfassembles into a long helical structure when expressed in Escherichia coli. However, the NPqe 375 subjects to proteolytic activity of host cell endogenous proteases during the protein recovery process. Image analysis of Western blots using the Quantity One software was performed to identify the size of the degraded bands and hence the potential proteases cleavage sites were predicted. The data obtained from this image analysis were compared to those identified with the PeptideCutter program; the potential proteases that degrade the NPqe 375 were identified to be mainly the metallo and serine proteases. Combination of ethylenediaminetetraacetic acid and phenylmethylsulfonyl fluoride at their optimal concentration gave a synergistic effect and increased the NPqe 375 yield by 2.1 -fold. The antigenicity and self-assembled long helical structure long helical structure of NPqe375 were preserved after treatment with the protease inhibitors.
\end{abstract}

Keyword: Endogenous protease; Escherichia coli; Newcastle disease virus; Nucleocapsid protein; PeptideCutter; Protease inhibitor 